\title{
Expanding The Frontier One Asset at a Time
}

\author{
Andrey D. Ukhov* \\ Indiana University
}

May 12, 2005

\begin{abstract}
We study the mean-variance optimization problem when investment opportunities are changing. We add a new risky asset to a set of $n$ risky assets. An analytical relation between the original and the new minimum-variance frontiers is established. The two frontiers have a tangency point. We derive a new mutual fund theorem. All portfolios in the new minimum-variance set are portfolio combinations of three mutual funds: The two funds located on the original frontier and the third fund containing all assets. Analytical framework developed in the paper has implications for studies of testability of the mean-variance efficiency of a market portfolio (Roll critique). Implications for models of financial innovation are discussed.
\end{abstract}

(Mean-variance Analysis; Efficient frontier; Mutual fund separation theorem; Roll critique; Financial innovation)

${ }^{*}$ I thank William N. Goetzmann, Jonathan E. Ingersoll, Jr., and Matthew Spiegel for helpful comments on an earlier draft of the paper. A part of this research was conducted when I was a Doctoral Fellow at the International Center for Finance at Yale School of Management. Address: Andrey D. Ukhov, Kelley School of Business, Indiana University, 1309 East Tenth Street, Bloomington, IN 47405. Tel. 812-855-2698. E-mail: aukhov@indiana.edu 


\title{
Expanding The Frontier One Asset at a Time
}

\begin{abstract}
We study the mean-variance optimization problem when investment opportunities are changing. We add a new risky asset to a set of $n$ risky assets. An analytical relation between the original and the new minimum-variance frontiers is established. The two frontiers have a tangency point. We derive a new mutual fund theorem. All portfolios in the new minimum-variance set are portfolio combinations of three mutual funds: The two funds located on the original frontier and the third fund containing all assets. Analytical framework developed in the paper has implications for studies of testability of the mean-variance efficiency of a market portfolio (Roll critique). Implications for models of financial innovation are discussed.
\end{abstract}




\section{Introduction}

The mean-variance portfolio analysis pioneered by Markowitz (1952) lies at the foundation of the modern portfolio theory. Properties of the minimum-variance frontier have been studied by many authors (Markowitz 1952, 1991; Merton 1972; Dybvig 1984; Huberman and Kandel 1987; Ingersoll 1987; and most recently by Feldman and Reisman 2003). ${ }^{1}$

Mean-variance analysis is a static framework. The number of risky assets and the asset characteristics are fixed. The reality of the world's financial markets is quite different. The investment opportunity set changes. New assets are introduced through several channels, including initial public offerings (IPOs), privatization programs, and financial market liberalizations, to name a few. An important issue, therefore, is how the efficient portfolios change when we introduce a new security and change the market structure of the economy. What is the relation between the mean-variance frontier generated with the original assets and the new frontier generated by the set of assets that includes the original securities and a new asset? What are the important characteristics of the investment opportunity set that determine their relation? Our paper addresses these questions.

In this paper we study how the mean-variance frontier changes when a new risky asset is added to the set of existing risky assets. We begin with a set of $n$ risky assets. It is well known that the equation of the minimum variance set is a parabola in mean-variance space. We keep the original assets and add a new risky asset. The new investment opportunity set contains $(n+1)$ assets. In this case, too, the equation of the minimum variance set is a parabola. Equations of the original and the new parabola each contain three coefficients. We derive an analytic relation between the

\footnotetext{
${ }^{1}$ Merton (1972) develops an analytic derivation of the efficient portfolio frontier. Dybvig (1984) studies the impact of a short sale restriction on the geometry of the frontier. Huberman and Kandel (1987) study spanning in a setting where they restric returns on $K+N$ risky assets to be generated by a linear model with $K$ factors. Notably, the analysis of spanning in our paper does not rely on the assumption of a linear factor model. Feldman and Reisman (2003) develop and elegant construction of the frontier. Practitioners, too, continue to retain strong interest in the method. It is interesting to note that a quick search of patents issued in the United States reveals several recent patents on the topic of portfolio formation (Michaud and Michaud 1999; Giansante and Dragun 2001; Scott et al. 2001). For the relation between behavioral finance the mean-variance paradigm see Shefrin and Statman (1994; 2000), and Levy and Levy (2004).
} 
corresponding coefficients of the two parabolas. We show that the expected return of the new asset affects the relation between two out of three parabola coefficient pairs. We also show that relations between all parabola coefficient pairs are affected by the vector of covariances of the new asset with all original assets. We show that the new mean-variance set generated with the original $n$ assets and the new asset, and the old set (generated with the original $n$ assets only) have a point of tangency. This is the point on the new set where no wealth is invested in the new asset.

One of the central results in mean variance analysis is the mutual fund separation theorem. This theorem states that given $n$ risky assets, there are two portfolios (mutual funds) constructed from these $n$ assets that any portfolio on the minimum variance frontier can be attained as a linear combination of these two mutual funds. In the case of the changing asset set, we extend this result to a Three Mutual Fund Theorem. We show that all portfolios on the new frontier are portfolio combinations of only three mutual funds. Two of these mutual funds invest in the original $n$ assets only, and therefore span the original minimum variance set. The third mutual fund has non-zero wealth invested in the new asset. Generally, the third mutual fund invests in all assets. This separation result highlights the importance of the covariance structure. The theorem shows that, in general, when a new asset becomes available, an investor cannot simply sell some shares in each of the two mutual funds that span his original portfolio allocation and use the proceeds to buy the new asset. Investing in the new asset and in the two mutual funds that span the original frontier does not span the new frontier. A third mutual fund is needed. This fund invests in all assets in the economy. With the introduction of the third fund the investor allocates wealth between the two original funds and the new fund. The theorem implies that when a new asset becomes available we can expect a non-trivial amount of re-balancing to take place in the economy. There is one case when the mutual fund problem above simplifies. This is the case when the new asset is not correlated with any of the existing assets. Then the third mutual fund needed to span the new frontier is simply the new asset. This analysis shows how the covariance of the new asset with existing assets affects spanning.

The effect of the changing asset set on the mean-variance frontier can be studied from several perspectives. The first approach is focused on the sensitivity of MV analysis to the inputs. The 
second interesting aspect of the problem has been investigated in connection with empirical studies of the Capital Asset Pricing Model because of exclusion of assets, or even asset classes, from the market portfolio proxies. The third perspective is due to the literature on financial innovation. We briefly discuss all three.

It has been established that the performance of optimal mean-variance portfolios is sensitive to the risk and return characteristics of the assets included in the investment opportunity set (Chopra and Ziemba (1993)). A mean-variance-efficient portfolio's weights, mean, and variance can be very sensitive to changes in asset means (Best and Grauer (1991)). ${ }^{2}$ Empirical researchers have long argued that changes in parameters affect tests of the CAPM, a theory that is based on MV analysis. ${ }^{3}$ Changes in the composition of assets can be expected to present additional challenges. ${ }^{4}$ In his fundamental critique of the asset pricing theory tests, Roll (1977) argues that CAPM “...is not testable unless the exact composition of the market portfolio is known and used in the tests." Exclusion of assets can have a significant effect on the location of the market portfolio in the meanvariance space, and therefore a large impact on the results of empirical tests. Stambaugh (1982) investigates the sensitivity of tests of the CAPM to different sets of asset returns. Kandel (1984) and Shanken (1986) also study this problem and conclude that the testability of the mean variance efficiency of a market index is problematic, when the returns on some components of the index are not perfectly observable. Our results are consistent with these findings. Our main theorem proves the importance of covariances of the return on the missing asset with the returns on included assets. The main theorem shows that knowing the expected return and the variance of the missing asset is not enough for spanning the frontier of all assets and for locating the market portfolio.

Literature on financial innovation investigates the effect of changing investment opportunities

\footnotetext{
${ }^{2}$ The sensitivity of the optimal portfolio allocation to inputs was also investigated in Pastor and Stambaugh (2000). Ferreira and Gama (2005) discuss changes in variances and correlations between countries and industries and implications for global portfolio management.

${ }^{3}$ Engle and Rodrigues (1987); Gibbons and Ferson (1985); Ferson, Kandel, and Stambaugh (1987).

${ }^{4}$ See Stambaugh (1982), Kandel (1984), and Shanken (1986). Gibbons, Ross, and Shanken (1989) develop a test for whether any particular portfolio is ex ante mean-variance efficient. They also study the sensitivity of the test to the number of assets used to determine the ex post mean-variance frontier.
} 
from a theoretical standpoint. As a new security (or an asset class) enters the investment opportunity set, it changes the risk-sharing structure of the economy (Allen and Gale (1994)). This can lead to changes in asset prices. The results, however, depend on the economic model. Oh (1996) studies the effects of introduction of new assets on asset prices within CAPM framework. He proves that financial innovation does not affect the price of any risky security relative to the bond in standard mean-variance setting. Our analysis, however, shows that even within the mean-variance framework financial innovation has important impact on portfolio rebalancing and therefore on trading volume. The model that captures these effects is yet to be developed.

The paper is structured as follows. In the next section we review the main results of the meanvariance portfolio selection. In section 3 we study the problem of adding a new asset to a set of existing assets. We derive the relation between the inverse of the covariance matrix for the $(n+1)$ and $n$-asset cases in 3.1. The relation between two frontiers is studied in 3.2. The mutual fund theorem (main result) is proven in 3.3. Section 4 concludes.

\section{Standard Mean-Variance Portfolio Problem}

We work within the framework of the standard mean-variance portfolio problem. In this section we review several results due to Merton (1972). ${ }^{5}$ For convenience, we keep the notation consistent with the classical reference, Ingersoll (1987). We begin with $n$ risky assets with nonsingular covariance matrix $\Sigma_{n}$. We also assume that all assets do not have the same mean, $\overline{\mathbf{z}}_{n} \neq k \mathbf{1}_{n}$, where $\mathbf{1}_{n}$ is the unit vector of length $n$. Vector $\overline{\mathbf{z}}_{n}$ is the vector of expected returns (one plus the rate of return) on the risky assets. We use subscript to indicate the number of risky assets in the problem.

The minimum-variance portfolio with expected return $\mu_{n}$ is the solution $\mathbf{w}_{n}\left(\mu_{n}\right)$ to

$$
\begin{gathered}
\min \frac{1}{2} \mathbf{w}_{n}^{\prime} \Sigma_{n} \mathbf{w}_{n} \\
\text { Subject to } \mathbf{1}_{n}^{\prime} \mathbf{w}_{n}=1, \\
\overline{\mathbf{z}}_{n}^{\prime} \mathbf{w}_{n}=\mu_{n} .
\end{gathered}
$$

\footnotetext{
${ }^{5}$ See also the intuitive derivation in Feldman and Reisman (2003).
} 
The Lagrangian is given by $L \equiv \mathbf{w}_{n}^{\prime} \Sigma_{n} \mathbf{w}_{n} / 2+\lambda_{n}\left(1-\mathbf{1}_{n}^{\prime} \mathbf{w}_{n}\right)+\gamma_{n}\left(\mu_{n}-\overline{\mathbf{z}}_{n}^{\prime} \mathbf{w}_{n}\right)$. Differentiating gives the first-order conditions, from which we obtain the solution set

$$
\begin{aligned}
\mathbf{w}_{n}^{*} & =\lambda_{n} \Sigma_{n}^{-1} \mathbf{1}_{n}+\gamma_{n} \Sigma_{n}^{-1} \overline{\mathbf{z}}_{n}, \\
\lambda_{n} & =\frac{C_{n}-\mu_{n} B_{n}}{\triangle_{n}}, \quad \gamma_{n}=\frac{\mu_{n} A_{n}-B_{n}}{\triangle_{n}}, \\
A_{n} & =\mathbf{1}_{n}^{\prime} \Sigma_{n}^{-1} \mathbf{1}_{n}>0, \quad B_{n}=\mathbf{1}_{n}^{\prime} \Sigma_{n}^{-1} \overline{\mathbf{z}}_{n}, \\
C_{n} & =\overline{\mathbf{z}}_{n}^{\prime} \Sigma_{n}^{-1} \overline{\mathbf{z}}_{n}>0, \quad \triangle_{n}=A_{n} C_{n}-\left(B_{n}\right)^{2}>0 .
\end{aligned}
$$

The equation of the minimum variance set is

$$
\sigma_{n}^{2}=\frac{A_{n} \mu_{n}^{2}-2 B_{n} \mu_{n}+C_{n}}{\triangle_{n}}
$$

which is the equation of a parabola in mean-variance space.

The Global Minimum Variance (GMV) portfolio is a special portfolio. It has the lowest variance among all possible portfolios. Asset weights in the GMV portfolio depend only on the covariance matrix and do not depend on asset expected returns. When there are $n$ risky assets the vector of GMV portfolio weights is given by

$$
\mathbf{w}_{n g}=\left[\Sigma_{n}^{-1} \mathbf{1}_{n}\right] / A_{n}=\left[\Sigma_{n}^{-1} \mathbf{1}_{n}\right] /\left[\mathbf{1}_{n}^{\prime} \Sigma_{n}^{-1} \mathbf{1}_{n}\right]
$$

The Global Minimum Portfolio has an expected return of $B_{n} / A_{n}$ and a variance of $1 / A_{n}$.

\section{Changing Asset Set}

Let $\mathcal{V}_{n}$ denote the set of minimum variance portfolios obtained from the set of $n$ risky assets. Let $p \in \mathcal{V}_{n}$ be a portfolio in this set. Then $\mathbf{w}_{n p}$ is $n$-vector of weights of the risky assets in the portfolio $p, \mu_{p} \equiv \mathbb{E}\left[\widetilde{r}_{p}\right]$ is the expected rate of return on the portfolio, and $\sigma_{p}^{2} \equiv \operatorname{Var}\left[\widetilde{r}_{p}\right]$ is the variance of the portfolio return. Portfolio $p$ is uniquely characterized by its expected return, $\mu_{p}$, because portfolio weights $\mathbf{w}_{n p}$ are determined from the mean-variance optimization problem for a given $\mu_{p}$. The converse is also true: the vector of portfolio weights $\mathbf{w}_{n p}$ uniquely characterizes (determines) portfolio $p$. 
We keep the original $n$ assets and add another risky asset, indexed by subscript $a$. This asset is not part of the original set of risky assets and it was not used in the construction of the set $\mathcal{V}_{n}$. This asset is characterized by its expected return, $\mu_{a} \equiv \mathbb{E}\left[\widetilde{r}_{a}\right], n$-vector of covariances with the original $n$ risky assets: $\boldsymbol{\sigma}_{n a}=\left(\sigma_{1 a}, \sigma_{2 a}, \ldots, \sigma_{n a}\right)^{\prime}$, where $\sigma_{i a}=\operatorname{Cov}\left[\widetilde{r}_{i}, \widetilde{r}_{a}\right]$ for $i=1, \ldots, n$ and its variance, $\sigma_{a}^{2}=\operatorname{Var}\left[\widetilde{r}_{a}\right]$.

When a new asset is added to the original set of $n$ risky assets the covariance matrix becomes

$$
\Sigma_{(n+1)}\left(\begin{array}{c}
\sigma_{1 a} \\
\vdots \\
\sigma_{n a} \\
\sigma_{n}
\end{array}\right)=\left(\begin{array}{cc}
\Sigma_{n} & \boldsymbol{\sigma}_{n a} \\
\boldsymbol{\sigma}_{n a}^{\prime} & \sigma_{a}^{2}
\end{array}\right),
$$

where $\boldsymbol{\sigma}_{n a}$ is a column vector. When changing from $n$ to $(n+1)$ assets we also modify the vector of expected returns:

$$
\overline{\mathbf{z}}_{(n+1)}=\left(\bar{z}_{1}, \ldots, \bar{z}_{n}, \bar{z}_{a}\right)^{\prime}
$$

The solution to the mean variance portfolio problem involves the computation of the inverse of the covariance matrix. Formulae for optimal portfolio weights (2), (3), (4a) and (4b), and for the minimum variance set (5) include the inverse of the covariance matrix. Therefore, if we aim at establishing a relation between portfolios in the $n$ and the $(n+1)$ asset cases, we need to study the changes to the covariance matrix and the inverse of the covariance matrix. We need to establish a link between the inverse of $\Sigma_{(n+1)}$ and the inverse of $\Sigma_{n}$. Several useful mathematical results on computing inverses of partitioned matrices are given in the Appendix. The following Lemma provides the foundation for the analysis of $\Sigma_{(n+1)}^{-1}$.

Lemma $1 \Sigma$ and $\Omega$ are two invertible matrices with dimension $(p, p)$ and $(n, n)$, respectively, and $\mathbf{V}$ is an $(n, p)$ matrix. Then the inverse of a partitioned matrix is given by

$$
\left(\begin{array}{ll}
\Sigma & \Sigma \mathbf{V}^{\prime} \\
\mathbf{V} \Sigma & \mathbf{V} \Sigma \mathbf{V}^{\prime}+\Omega
\end{array}\right)^{-1}=\left(\begin{array}{ll}
\Sigma^{-1}+\mathbf{V}^{\prime} \Omega^{-1} \mathbf{V} & -\mathbf{V}^{\prime} \Omega^{-1} \\
-\Omega^{-1} \mathbf{V} & \Omega^{-1}
\end{array}\right) .
$$

Proof. The proof (see Appendix) is by direct computation, using block multiplication rule. 


\subsection{The Relation Between $\Sigma_{n}^{-1}$ and $\Sigma_{(n+1)}^{-1}$}

We now derive a relationship between the covariance matrices of the $n$-asset and the $(n+1)$ asset case.

Proposition 2 The inverse of $\Sigma_{(n+1)}$ is related to the inverse of $\Sigma_{n}$ and to the parameters $\boldsymbol{\sigma}_{n a}$ and $\sigma_{a}^{2}$ as follows

$$
\Sigma_{(n+1)}^{-1}=\left(\begin{array}{cc}
\Sigma_{n} & \boldsymbol{\sigma}_{n a} \\
\boldsymbol{\sigma}_{n a}^{\prime} & \sigma_{a}^{2}
\end{array}\right)^{-1}=\left(\begin{array}{cc}
\Sigma_{n}^{-1}+\Sigma_{n}^{-1} \boldsymbol{\sigma}_{n a} \Omega^{-1} \boldsymbol{\sigma}_{n a}^{\prime} \Sigma_{n}^{-1} & -\Sigma_{n}^{-1} \boldsymbol{\sigma}_{n a} \Omega^{-1} \\
-\Omega^{-1} \boldsymbol{\sigma}_{n a}^{\prime} \Sigma_{n}^{-1} & \Omega^{-1}
\end{array}\right)
$$

where $\Omega=\sigma_{a}^{2}-\boldsymbol{\sigma}_{n a}^{\prime} \Sigma_{n}^{-1} \boldsymbol{\sigma}_{n a}$, and $\Omega \in \mathbb{R}$.

Proof. The proof (see Appendix) consists of two steps. First, we establish a correspondence between the variables in the Lemma $1, \Sigma, \mathbf{V}, \Omega$, and the components of the $\Sigma_{(n+1)}$ matrix. We then apply Lemma 1.

\subsection{The Relation Between Two Frontiers}

Having established the relation between $\Sigma_{(n+1)}^{-1}$ and $\Sigma_{n}^{-1}$, we proceed to analyze the relation between two minimum variance sets. It follows from (5) that each minimum variance set is described by three numbers, $A_{(n+1)}, B_{(n+1)}, C_{(n+1)}$, and $A_{n}, B_{n}, C_{n}$, respectively. The following proposition establishes the relation between the parameters that define the minimum variance sets.

Proposition 3 As before, define $\Omega \in \mathbb{R}$, and also define $D, E \in \mathbb{R}$,

$$
\Omega \equiv \sigma_{a}^{2}-\boldsymbol{\sigma}_{n a}^{\prime} \Sigma_{n}^{-1} \boldsymbol{\sigma}_{n a}, \quad D \equiv-\Omega^{-1} \boldsymbol{\sigma}_{n a}^{\prime} \Sigma_{n}^{-1} \mathbf{1}_{n}, \quad E \equiv-\Omega^{-1} \boldsymbol{\sigma}_{n a}^{\prime} \Sigma_{n}^{-1} \overline{\mathbf{z}}_{n} .
$$

Then

$$
\begin{aligned}
& A_{(n+1)}=A_{n}+[1+D \Omega]\left[D+\Omega^{-1}\right], \\
& B_{(n+1)}=B_{n}+[1+D \Omega]\left[E+\Omega^{-1} \bar{z}_{a}\right], \\
& C_{(n+1)}=C_{n}+\left[E \Omega+\bar{z}_{a}\right]\left[E+\Omega^{-1} \bar{z}_{a}\right] .
\end{aligned}
$$


Coefficients of the parabola (in mean-variance space) constructed with $n+1$ securities are related to the coefficients of the parabola created with $n$ securities. The relation between the parabolas depends on the expected return on the new asset. The expected return affects the relation between parabola coefficients $B_{(n+1)}$ and $B_{n}$, and between $C_{(n+1)}$ and $C_{n}$. The expected return does not affect the relation between $A_{(n+1)}$ and $A_{n}$. The relations between all parabola coefficients depend on the covariance structure captured by the inverse of the covariance matrix, $\Sigma_{n}^{-1}$. The relations also depend on the variance of the new asset, $\sigma_{a}^{2}$, and on the covariances of the new asset with all existing assets in the economy-the vector of covariances, $\boldsymbol{\sigma}_{n a}$.

It is a well-known property of the portfolio problem that for each asset there is one minimumvariance portfolio in which it has a weight of zero. Therefore, on the frontier constructed with $n+1$ assets there will be one point that has a weight of zero for the new asset. This point uses only the $n$ original assets. This point, then, also lies on the frontier generated with the original $n$ assets. This proves the following result.

Proposition 4 The new minimum-variance set generated with the original $n$ assets and the new asset, and the old minimum-variance set (generated with the original $n$ assets only) have a point of tangency. This is the point where no wealth is invested in the new $(n+1)$-st asset.

The point of tangency of the two frontiers may be located above the $G M V_{n}$ portfolio (and above the $G M V_{(n+1)}$ portfolio). This case is shown on Figure 1. In this case, $G M V_{(n+1)}$ is located below the $G M V_{n}$. The expected return on $G M V_{(n+1)}$ is less than the expected return on $G M V_{n}$,

$$
\frac{B_{(n+1)}}{A_{(n+1)}}<\frac{B_{n}}{A_{n}}
$$

The tangency point may also be located below the $G M V_{n}$ portfolio (and below the $G M V_{(n+1)}$ portfolio). This case is shown on Figure 2. In this case the $G M V_{(n+1)}$ portfolio is located above the $G M V_{n}$. The expected return on $G M V_{(n+1)}$ is higher than the expected return on $G M V_{n}$,

$$
\frac{B_{(n+1)}}{A_{(n+1)}}>\frac{B_{n}}{A_{n}}
$$




\subsection{A Mutual Fund Theorem}

The separation theorem, or the mutual fund theorem, is one of the most important results in mean variance analysis (Merton 1972; Ingersoll 1987). ${ }^{6}$ When studying the efficient frontier, Merton (1972) established the following result.

Proposition 5 (Theorem 1 in Merton 1972) Given $n$ risky assets, there are two portfolios ("mutual funds") constructed from these $n$ assets, such that all risk-averse individuals, who choose their portfolios so as to maximize utility functions dependent only on the mean and variance of their portfolios, will be indifferent in choosing between portfolios from among the original $n$ assets or from these two funds.

Corollary 6 Given the mutual fund theorem, and the two mutual funds constructed from $n$ assets, the investor need only know the means, variances, and covariances of the two funds to determine the mix of the two funds that generates his optimal portfolio.

To prove the theorem, Merton (1972) shows how any portfolio on the efficient frontier can be attained by a linear combination of two distinct portfolios. From (2) we can see that all minimum variance portfolios are portfolio combinations of only two distinct portfolios. The global minimumvariance portfolio corresponds to the first term in (2). GMV portfolio weights, $\mathbf{w}_{n g}$, are given in (6). Define the second portfolio,

$$
\mathbf{w}_{n d}=\left[\Sigma_{n}^{-1} \overline{\mathbf{z}}_{n}\right] / B_{n}=\left[\Sigma_{n}^{-1} \overline{\mathbf{z}}_{n}\right] /\left[\mathbf{1}_{n}^{\prime} \Sigma_{n}^{-1} \overline{\mathbf{z}}_{n}\right]
$$

and write (2) as

$$
\mathbf{w}_{n}^{*}=\left(\lambda_{n} A_{n}\right) \mathbf{w}_{n g}+\left(\gamma_{n} B_{n}\right) \mathbf{w}_{n d}
$$

Since $\left(\lambda_{n} A_{n}\right)+\left(\gamma_{n} B_{n}\right)=1$, we have a portfolio of two mutual funds.

The two mutual fund theorem applies in each setting. It applies to the set $\mathcal{V}_{n}$ generated by the original $n$ assets. There are two mutual funds, each being a combination of the $n$ assets, that span

\footnotetext{
${ }^{6}$ For a general discussion of mutual fund or "separation" theorems, see Cass and Stiglitz (1970).
} 
the frontier. The theorem also applies to the case of $(n+1)$ assets. For the set $\mathcal{V}_{(n+1)}$ generated by the original $n$ assets and the new asset $a$, there are also two mutual funds, each being a combination of $n+1$ assets, that span the new frontier. Generally speaking, the first two mutual funds and the second two mutual funds need not be the same. There is an important connection, however, that we establish below.

In the main theorem of the paper (below), we show that all portfolios in $\mathcal{V}_{(n+1)}$ are portfolio combinations of only three mutual funds. Two of these mutual funds span the original minimumvariance set, $\mathcal{V}_{n}$. These two portfolios invest only in the original $n$ assets. The third portfolio has non-zero wealth invested into the new risky asset.

Theorem 7 (Main Result: Three Mutual Fund Theorem) Given the "original" $n$ risky assets and the new risky asset, the "three mutual fund" separation holds. There are two mutual funds constructed with the original $n$ assets only and one mutual fund constructed with all $n+1$ assets. All risk-averse individuals, who choose their portfolios so as to maximize utility functions dependent only on the mean and variance of their portfolios, will be indifferent in choosing between portfolios from among the $n+1$ assets or from these three funds. In other words, the mean-variance frontier constructed with $n+1$ assets is spanned by three mutual funds. Two of the mutual funds are located on the original frontier (constructed with $n$ assets only). Optimal portfolio weights in the $n+1$ case are given by

$$
\begin{aligned}
\mathbf{w}_{(n+1)}^{*}(\mu)= & \lambda_{(n+1)} A_{n}\left[\begin{array}{l}
\mathbf{w}_{n g} \\
0
\end{array}\right]+\gamma_{(n+1)} B_{n}\left[\begin{array}{l}
\mathbf{w}_{n d} \\
0
\end{array}\right] \\
& +(D \Omega+1)\left(\lambda_{(n+1)}\left(D+\Omega^{-1}\right)+\gamma_{(n+1)}\left(E+\Omega^{-1} \bar{z}_{a}\right)\right)\left[\begin{array}{l}
-\Sigma_{n}^{-1} \boldsymbol{\sigma}_{n a} /(D \Omega+1) \\
1 /(D \Omega+1)
\end{array}\right] .
\end{aligned}
$$

Proof. See Appendix.

Corollary $\mathbf{8}$ The portfolio weight of the new asset is given by

$$
w_{a}^{*}(\mu)=\lambda_{(n+1)}\left(D+\Omega^{-1}\right)+\gamma_{(n+1)}\left(E+\Omega^{-1} \bar{z}_{a}\right) .
$$


The intuition behind the theorem is based on two facts. First, each of the frontiers is spanned by two portfolios. Second, the two frontiers have a tangency point. We know that any two portfolios in $\mathcal{V}_{(n+1)}$ span $\mathcal{V}_{(n+1)}$. We also know that $\mathcal{V}_{(n+1)}$ and $\mathcal{V}_{n}$ have a tangency point. Pick this tangency point as one of the two portfolios that $\operatorname{span} \mathcal{V}_{(n+1)}$. Further, since this tangency portfolio is also in $\mathcal{V}_{n}$, it is spanned by any two portfolios in $\mathcal{V}_{n}$. So we have three portfolios: two in $\mathcal{V}_{n}$ that are combined to give us the tangency portfolio and one more portfolio from $\mathcal{V}_{(n+1)}$. Since the first two portfolios are in $\mathcal{V}_{n}$, they do not invest in the new asset.

One important implication of the theorem is for portfolio rebalancing. Consider an investor who holds an optimal portfolio constructed with the original $n$ assets. Then a new asset becomes available. The theorem shows that the investor cannot simply sell some shares in each of the two mutual funds and use the proceeds to buy the new asset. Investing in the new asset and in the two mutual funds that span the original frontier does not span the new frontier. What is needed is a third mutual fund that invests in all assets in the economy. With the third fund, the investor re-allocates between the two original funds and the new fund. The introduction of a new asset causes re-balancing because a new mutual fund needs to be created.

Let us consider a special case when the new asset is not correlated with the existing assets, $\boldsymbol{\sigma}_{n a}=\mathbf{0}$. In this case $\Omega=\sigma_{a}^{2}, D=E=0$. Portfolio weights are given by

$$
\mathbf{w}_{(n+1)}^{*}(\mu)=\lambda_{(n+1)} A_{n}\left[\begin{array}{l}
\mathbf{w}_{n g} \\
0
\end{array}\right]+\gamma_{(n+1)} B_{n}\left[\begin{array}{l}
\mathbf{w}_{n d} \\
0
\end{array}\right]+\left(\frac{\lambda_{(n+1)}}{\sigma_{a}^{2}}+\frac{\gamma_{(n+1)} \bar{z}_{a}}{\sigma_{a}^{2}}\right)\left[\begin{array}{l}
\mathbf{0} \\
1
\end{array}\right] .
$$

In this case the third mutual fund needed to span the new frontier is simply the new asset. If an asset is introduced that is uncorrelated with all existing assets, then rebalancing is simple. Any mean-variance optimizing investors sells some of his holdings in both existing mutual funds. He invests the proceeds into the new asset. 


\section{Discussion and Conclusion}

\subsection{The Importance of Covariances}

Proposition 3 shows that the relation between the efficient frontier constructed with $n$ risky assets and the frontier constructed with $(n+1)$ assets critically depends on the covariance structure. The vector of covariances of the new asset with the existing assets is one of the important determinants of the relation.

Another new result in our paper is the three mutual fund theorem that shows the connection between the classic mean-variance two mutual fund spanning result and the role of co-variances. When the new asset is uncorrelated with all existing assets, the new frontier is spanned by the new asset and by two mutual funds located on the old frontier. When the new asset is correlated with any of the existing assets, the picture changes significantly. The new frontier can no longer be spanned by the investment in the new asset alone (and the two mutual funds containing only the old assets). In addition to the two funds that invest in the original securities only, we now need a third fund that holds all $(n+1)$ assets.

The notion that $c o$-movement in asset returns matters in the context of the portfolio problem and international diversification was understood by British investors nearly one hundred years ago. $^{7}$ The quantitative analysis of international diversification dates at least to Henry Lowenfeld's (1907) study of equal-weighted, industry-neutral, international diversification strategies. In his book, he argues that superior investment performance can be obtained by spreading capital in equal proportion across a number of geographical areas and carefully re-balancing back to these proportions on a regular basis. To support the recommendation for international diversification, Lowenfeld provides charts of price movements of the stocks in the following countries: Great Britain, France, Germany, Switzerland, Belgium, Canada, Japan, USA, and Argentina. He concludes that the value of all securities that come from the same country is influenced by that country's market factor. At the same time, he points out that securities from different countries do not have the same degree of co-movement as securities within one country. This observation allows Lowenfeld

\footnotetext{
${ }^{7}$ Goetzmann and Ukhov(2005).
} 
to put forward the investment principle which he calls "The Geographical Distribution of Capital." According to this principle, an investor spreads his wealth across securities from different countries, thereby diversifying away country-specific risks.

The importance of covariances has been shown in the studies of the relationship between the number of securities in a portfolio and portfolio risk. ${ }^{8}$ Fama (1976, Chapter 7 ) shows that the contribution of a security to the risk of a portfolio depends on the covariances between the return on the security and the returns on other assets in the portfolio, as well as on the variance of the return on the security. In a portfolio where a relatively small fraction of wealth is invested in an asset, the contribution of this asset to portfolio risk might depend more on the covariances than on the variance of the security's return. Similar intuition is developed in Elton and Gruber (1977) who show (assuming equal portfolio weights) that as the number of securities in a portfolio increases, portfolio variance is determined by the average covariance between the assets in the portfolio, and not by the average variance of the securities.

\subsection{Financial Innovation}

Financial innovation changes the investment opportunity set. Introduction of new securities changes the menu of payoffs in different states of nature that are available to investors for purchase. In this case, risk-sharing opportunities in the economy change, too. Investors may be able to hedge risks better when a new asset or an asset class becomes available (Allen and Gale (1994), Miller (1991)). Changes in risk-sharing cause changes in demand for the risky assets that can lead to changes in asset prices. The results depend on the equilibrium model deployed. Oh (1996) shows that financial innovation does not affect risk pricing in standard mean-variance settings when the set of agents in the economy is fixed. Specifically in quadratic or CARA-normal economies, the price of any risky security relative to the bond is unaffected by changes in the span. This result may explain why researchers have made little use of mean-variance models to analyze the effect of

\footnotetext{
${ }^{8}$ See Fama (1976), Chapter 7; Elton and Gruber (1977), and Statman (1987).
} 
financial innovation on asset prices. ${ }^{9}$ The Three Mutual Fund Theorem shows that within meanvariance framework financial innovation can have important implications for portfolio rebalancing and trading volume.

\subsection{Empirical Tests of the CAPM}

Our main theorem has implications for the literature on testability of the mean-variance efficiency of a market portfolio when characteristics (return, variance, covariance) of some component of the portfolio are not perfectly observable (Stambaugh (1982), Kandel (1984), Shanken (1986)). In his famous critique of the asset pricing theory tests, Roll (1977) argues that CAPM “...is not testable unless the exact composition of the market portfolio is known and used in the tests. This implies that the theory is not testable unless all individual assets are included in the sample. ...even a small misspecification of the proxy's composition can lead to the wrong conclusion." (The market proxy might be mean-variance efficient while the true market portfolio is not, and vice versa.) ${ }^{10}$ Stambaugh (1982) investigates whether the tests of the CAPM are sensitive to different sets of asset returns. He finds that inferences are sensitive to the set of assets used in the tests. Kandel (1984) studies the testability of the mean variance efficiency of a market index, when the returns on some components of the index itself are not perfectly observable. Only partial information is available about the parameters of the missing asset. The main finding in Kandel (1984) is that if there is no information about the missing asset, then the hypothesis of the mean variance efficiency of a portfolio cannot be rejected. He concludes that "the efficiency or inefficiency of a portfolio with respect to a subset of assets-by itself-tells us nothing about the efficiency of a larger portfolio." The same result holds even if we have perfect or partial information about the expected return of the missing asset. Kandel (1984) remarks that "it is interesting that the results highlight the importance of the variance of the missing asset."

Shanken (1986) includes risk-free asset in the analysis and addresses the question of whether

\footnotetext{
${ }^{9}$ Ukhov (2003) shows that in a model with endogenous stock market participation, introduction of a new asset can have an impact on prices of existing assets even in a CARA-Normal economy.

${ }^{10}$ See also Roll (1978).
} 
the unobservable market portfolio of risky assets is equal to the tangency portfolio of the efficient frontier. He finds that knowledge of the market share and expected return of the missing asset is still not sufficient to permit a test of this more restrictive efficiency condition. Our results are consistent with those of Kandel (1984) and Shanken (1986). Our analysis highlights the importance of covariances of the missing asset with the returns on included assets. We show that knowing the expected return and the variance of the missing asset is not enough for spanning the frontier of all assets. The results in Kandel (1984) and Shanken (1986) show the difficulties with spanning the frontier; we identify the role of unknown covariances in this problem.

\section{A Appendix}

There are several useful results on computing the inverse of a partitioned matrix. Let $\mathbf{M}$ be a square matrix of size $m$ partitioned as

$$
\mathbf{M}=\left(\begin{array}{ll}
\mathbf{M}_{11} & \mathbf{M}_{12} \\
\mathbf{M}_{21} & \mathbf{M}_{22}
\end{array}\right)
$$

where $\mathbf{M}_{11}, \mathbf{M}_{12}, \mathbf{M}_{21}$, and $\mathbf{M}_{22}$ are respectively of size $m_{1} \times m_{1}, m_{1} \times m_{2}, m_{2} \times m_{1}$, and $m_{2} \times m_{2}$ with $m_{1}+m_{2}=m$. Assume that $\mathbf{M}$ is nonsingular. Then its inverse matrix $\mathbf{M}^{-1}$ exists and it can be partitioned as

$$
\mathbf{M}^{-1}=\left(\begin{array}{ll}
\mathbf{M}^{11} & \mathbf{M}^{12} \\
\mathbf{M}^{21} & \mathbf{M}^{22}
\end{array}\right)
$$

where $\mathbf{M}^{11}, \mathbf{M}^{12}, \mathbf{M}^{21}$, and $\mathbf{M}^{22}$ are respectively of size $m_{1} \times m_{1}, m_{1} \times m_{2}, m_{2} \times m_{1}$, and $m_{2} \times m_{2}$ with $m_{1}+m_{2}=m$. We can determine $\mathbf{M}^{-1}$. To do this, we express the blocks $\mathbf{M}^{i j}$ of the inverse matrix in terms of the blocks $\mathbf{M}_{i j}$ of the original matrix. Then, use the matrix equality $\mathbf{M} \mathbf{M}^{-1}=\mathbf{I}$ and partition the identity matrix to obtain four equations in the four unknown matrices $\mathbf{M}^{11}, \mathbf{M}^{12}$, 
$\mathbf{M}^{21}$, and $\mathbf{M}^{22}$

$$
\begin{aligned}
& \mathbf{M}_{11} \mathbf{M}^{11}+\mathbf{M}_{12} \mathbf{M}^{21}=\mathbf{I} \\
& \mathbf{M}_{11} \mathbf{M}^{12}+\mathbf{M}_{12} \mathbf{M}^{22}=0 \\
& \mathbf{M}_{21} \mathbf{M}^{11}+\mathbf{M}_{22} \mathbf{M}^{21}=0 \\
& \mathbf{M}_{21} \mathbf{M}^{12}+\mathbf{M}_{22} \mathbf{M}^{22}=\mathbf{I}
\end{aligned}
$$

Assuming that $\mathbf{M}_{11}$ is nonsingular, from the first equation we obtain

$$
\mathbf{M}^{11}=\mathbf{M}_{11}^{-1}-\mathbf{M}_{11}^{-1} \mathbf{M}_{12} \mathbf{M}^{21}
$$

Substituting the last expression into the third equation and rearranging, we obtain

$$
\left(\mathbf{M}_{22}-\mathbf{M}_{21} \mathbf{M}_{11}^{-1} \mathbf{M}_{12}\right) \mathbf{M}^{21}=-\mathbf{M}_{21} \mathbf{M}_{11}^{-1}
$$

If $\mathbf{M}_{22}-\mathbf{M}_{21} \mathbf{M}_{11}^{-1} \mathbf{M}_{12}$ is nonsingular, then

$$
\begin{aligned}
& \mathbf{M}^{21}=-\left(\mathbf{M}_{22}-\mathbf{M}_{21} \mathbf{M}_{11}^{-1} \mathbf{M}_{12}\right)^{-1} \mathbf{M}_{21} \mathbf{M}_{11}^{-1}, \\
& \mathbf{M}^{11}=\mathbf{M}_{11}^{-1}+\mathbf{M}_{11}^{-1} \mathbf{M}_{12}\left(\mathbf{M}_{22}-\mathbf{M}_{21} \mathbf{M}_{11}^{-1} \mathbf{M}_{12}\right)^{-1} \mathbf{M}_{21} \mathbf{M}_{11}^{-1} .
\end{aligned}
$$

The remaining two blocks are computed from the other two equalities of the system. Then, for O defined as

$$
\mathbf{O} \equiv\left(\mathbf{M}_{22}-\mathbf{M}_{21} \mathbf{M}_{11}^{-1} \mathbf{M}_{12}\right)^{-1}
$$

we have the following result

$$
\mathbf{M}^{-1}=\left(\begin{array}{ll}
\mathbf{M}_{11}^{-1}+\mathbf{M}_{11}^{-1} \mathbf{M}_{12} \mathbf{O M}_{21} \mathbf{M}_{11}^{-1} & -\mathbf{M}_{11}^{-1} \mathbf{M}_{12} \mathbf{O} \\
-\mathbf{O M}_{21} \mathbf{M}_{11}^{-1} & \mathbf{O}
\end{array}\right)
$$

This is our first result for the inverse of a partitioned matrix. For future reference, it is convenient to formulate it as the following lemma. 
Lemma $\mathbf{9}$ Let $\mathbf{M}$ be a square matrix of size $m$ partitioned as

$$
\mathbf{M}=\left(\begin{array}{ll}
\mathbf{M}_{11} & \mathbf{M}_{12} \\
\mathbf{M}_{21} & \mathbf{M}_{22}
\end{array}\right)
$$

where $\mathbf{M}_{11}$ and $\mathbf{M}_{22}$ are square submatrices. If both $\mathbf{M}_{11}$ and the matrix $\mathbf{M}_{22}-\mathbf{M}_{21} \mathbf{M}_{11}^{-1} \mathbf{M}_{12}$ are nonsingular, then

$$
\mathbf{M}^{-1}=\left(\begin{array}{ll}
\mathbf{M}_{11}^{-1}+\mathbf{M}_{11}^{-1} \mathbf{M}_{12} \mathbf{O M}_{21} \mathbf{M}_{11}^{-1} & -\mathbf{M}_{11}^{-1} \mathbf{M}_{12} \mathbf{O} \\
-\mathbf{O M}_{21} \mathbf{M}_{11}^{-1} & \mathbf{O}
\end{array}\right)
$$

where $\mathbf{O} \equiv\left(\mathbf{M}_{22}-\mathbf{M}_{21} \mathbf{M}_{11}^{-1} \mathbf{M}_{12}\right)^{-1}$.

The following corollary states another useful relationship.

Corollary 10 The following equation holds true

$$
\begin{aligned}
& \mathbf{M}_{11}^{-1}+\mathbf{M}_{11}^{-1} \mathbf{M}_{12}\left(\mathbf{M}_{22}-\mathbf{M}_{21} \mathbf{M}_{11}^{-1} \mathbf{M}_{12}\right)^{-1} \mathbf{M}_{21} \mathbf{M}_{11}^{-1} \\
= & \left(\mathbf{M}_{11}-\mathbf{M}_{12} \mathbf{M}_{22}^{-1} \mathbf{M}_{21}\right)^{-1}
\end{aligned}
$$

Proof. The left-hand side of the equation equals $\mathbf{M}^{11}$, as was shown previously. The expression on the right-hand side also equals $\mathbf{M}^{11}$. To prove this, use the third equation of the system to obtain

$$
\mathbf{M}^{21}=-\mathbf{M}_{22}^{-1} \mathbf{M}_{21} \mathbf{M}^{11}
$$

and substitute into the first equation. Then

$$
\begin{aligned}
\mathbf{M}_{11} \mathbf{M}^{11}-\mathbf{M}_{12} \mathbf{M}_{22}^{-1} \mathbf{M}_{21} \mathbf{M}^{11} & =\mathbf{I} \\
\left(\mathbf{M}_{11}-\mathbf{M}_{12} \mathbf{M}_{22}^{-1} \mathbf{M}_{21}\right) \mathbf{M}^{11} & =\mathbf{I} \\
\left(\mathbf{M}_{11}-\mathbf{M}_{12} \mathbf{M}_{22}^{-1} \mathbf{M}_{21}\right)^{-1} & =\mathbf{M}^{11}
\end{aligned}
$$

The inverse of a partitioned matrix can be expressed in a somewhat different form. 
Lemma 11 Let $\mathbf{M}$ be a square matrix partitioned as

$$
\mathbf{M}=\left(\begin{array}{ll}
\mathbf{M}_{11} & \mathbf{M}_{12} \\
\mathbf{M}_{21} & \mathbf{M}_{22}
\end{array}\right)
$$

where $\mathbf{M}_{11}$ and $\mathbf{M}_{22}$ are square submatrices. If both $\mathbf{M}_{22}$ and the matrix

$$
\mathbf{Q}=\mathbf{M}_{11}-\mathbf{M}_{12} \mathbf{M}_{22}^{-1} \mathbf{M}_{21}
$$

are nonsingular, then $\mathbf{M}$ is nonsingular and

$$
\mathbf{M}^{-1}=\left(\begin{array}{ll}
\mathbf{Q}^{-1} & -\mathbf{Q}^{-1} \mathbf{M}_{12} \mathbf{M}_{22}^{-1} \\
-\mathbf{M}_{22}^{-1} \mathbf{M}_{21} \mathbf{Q}^{-1} & \mathbf{M}_{22}^{-1}\left(\mathbf{I}+\mathbf{M}_{21} \mathbf{Q}^{-1} \mathbf{M}_{12} \mathbf{M}_{22}^{-1}\right)
\end{array}\right)
$$

Proof. By direct computation:

$$
\begin{aligned}
\mathbf{M}^{-1} \times \mathbf{M} & =\left(\begin{array}{ll}
\mathbf{Q}^{-1} \mathbf{M}_{11}-\mathbf{Q}^{-1} \mathbf{M}_{12} \mathbf{M}_{22}^{-1} \mathbf{M}_{21} & \mathbf{Q}^{-1} \mathbf{M}_{12}-\mathbf{Q}^{-1} \mathbf{M}_{12} \mathbf{M}_{22}^{-1} \mathbf{M}_{22} \\
\mathbf{M}_{22}^{-1} \mathbf{M}_{21}\left(-\mathbf{Q}^{-1} \mathbf{Q}+\mathbf{I}\right) & \mathbf{I}
\end{array}\right) \\
& =\left(\begin{array}{ll}
\mathbf{Q}^{-1} \mathbf{Q} & \mathbf{Q}^{-1} \mathbf{M}_{12}-\mathbf{Q}^{-1} \mathbf{M}_{12} \\
\mathbf{0} & \mathbf{I}
\end{array}\right)=\left(\begin{array}{ll}
\mathbf{I} & \mathbf{0} \\
\mathbf{0} & \mathbf{I}
\end{array}\right) .
\end{aligned}
$$

Remark 12 The following is a reminder of well-known properties for computing inverses. Let $M$ and $O$ be square invertible matrices. Then, $\left(\mathbf{M}^{-1}\right)^{-1}=\mathbf{M},\left(\mathbf{M}^{T}\right)^{-1}=\left(M^{-1}\right)^{T}$, MO is invertible, and $(M O)^{-1}=O^{-1} M^{-1}$; for any scalar $r \neq 0, r M$ is invertible and $(r M)^{-1}=(1 / r) M^{-1}$.

Proof of Lemma 1. The proof is by direct computation, using the block multiplication rule.

$$
\begin{aligned}
& \left(\begin{array}{ll}
\Sigma & \Sigma \mathbf{V}^{\prime} \\
\mathbf{V} \Sigma & \mathbf{V} \Sigma \mathbf{V}^{\prime}+\Omega
\end{array}\right) \times\left(\begin{array}{cc}
\Sigma^{-1}+\mathbf{V}^{\prime} \Omega^{-1} \mathbf{V} & -\mathbf{V}^{\prime} \Omega^{-1} \\
-\Omega^{-1} \mathbf{V} & \Omega^{-1}
\end{array}\right) \\
= & \left(\begin{array}{ll}
\Sigma \Sigma^{-1}+\Sigma \mathbf{V}^{\prime} \Omega^{-1} \mathbf{V}-\Sigma \mathbf{V}^{\prime} \Omega^{-1} \mathbf{V} & -\Sigma \mathbf{V}^{\prime} \Omega^{-1}+\Sigma \mathbf{V}^{\prime} \Omega^{-1} \\
\mathbf{V} \Sigma \Sigma^{-1}+\mathbf{V} \Sigma \mathbf{V}^{\prime} \Omega^{-1} \mathbf{V}-\mathbf{V} \Sigma \mathbf{V}^{\prime} \Omega^{-1} \mathbf{V}-\Omega \Omega^{-1} \mathbf{V} & -\mathbf{V} \Sigma \mathbf{V}^{\prime} \Omega^{-1}+\mathbf{V} \Sigma \mathbf{V}^{\prime} \Omega^{-1}+\Omega \Omega^{-1}
\end{array}\right) \\
= & \left(\begin{array}{ll}
\mathbf{I}_{p \times p} & \mathbf{0} \\
\mathbf{0} & \mathbf{I}_{n \times n}
\end{array}\right)=\mathbf{I}_{(p+n) \times(p+n)},
\end{aligned}
$$


where $\mathbf{I}_{k \times k}$ is the $k \times k$ identity matrix.

Proof of Proposition 2. The proof consists of two steps. First, we establish a correspondence between the variables in the Lemma $1, \Sigma, \mathbf{V}, \Omega$, and the components of the $\Sigma_{(n+1)}$ matrix. We then apply Lemma 1 . The block $\Sigma$ is the covariance matrix $\Sigma_{n}$. The expression for $\mathbf{V}^{\prime}$ is found from $\Sigma_{n} \mathbf{V}^{\prime}=\boldsymbol{\sigma}_{n a}$. Note that $\mathbf{V}$ is a row vector, and, accordingly, $\mathbf{V}^{\prime}$ is a column vector. Since $\Sigma_{n}$ is invertible, $\mathbf{V}^{\prime}=\Sigma_{n}^{-1} \boldsymbol{\sigma}_{n a}$. Recall that for two matrices $A$ and $B,(A B)^{\top}=B^{\top} A^{\top}$ and deduce

$$
\mathbf{V}=\left(\mathbf{V}^{\prime}\right)^{\top}=\left(\Sigma_{n}^{-1} \boldsymbol{\sigma}_{n a}\right)^{\top}=\boldsymbol{\sigma}_{n a}^{\prime}\left(\Sigma_{n}^{-1}\right)^{\top}=\boldsymbol{\sigma}_{n a}^{\prime}\left(\Sigma_{n}^{\top}\right)^{-1}=\boldsymbol{\sigma}_{n a}^{\prime} \Sigma_{n}^{-1}
$$

where we used the standard property of a square invertible matrix, $\left(A^{\top}\right)^{-1}=\left(A^{-1}\right)^{\top}$, and relied on the symmetry of $\Sigma_{n}$.

Next, find $\Omega$ from

$$
\begin{aligned}
\sigma_{a}^{2} & =\mathbf{V} \Sigma_{n} \mathbf{V}^{\prime}+\Omega \\
& \Rightarrow \Omega=\sigma_{a}^{2}-\mathbf{V} \Sigma_{n} \mathbf{V}^{\prime}=\sigma_{a}^{2}-\boldsymbol{\sigma}_{n a}^{\prime} \Sigma_{n}^{-1} \Sigma_{n} \Sigma_{n}^{-1} \boldsymbol{\sigma}_{n a}=\sigma_{a}^{2}-\boldsymbol{\sigma}_{n a}^{\prime} \Sigma_{n}^{-1} \boldsymbol{\sigma}_{n a}
\end{aligned}
$$

Direct application of the Lemma to $\Sigma_{(n+1)}$ yields the proposition.

Proof of Proposition 3. The goal is to find the relation between $A_{(n+1)}$ and $A_{n}, B_{(n+1)}$ and $B_{n}, C_{(n+1)}$ and $C_{n}$. Work with $\Sigma_{(n+1)}^{-1} \mathbf{1}_{(n+1)}$

$$
\begin{aligned}
\Sigma_{(n+1)}^{-1} \mathbf{1}_{(n+1)} & =\left[\begin{array}{ll}
\Sigma_{n}^{-1}+\Sigma_{n}^{-1} \boldsymbol{\sigma}_{n a} \Omega^{-1} \boldsymbol{\sigma}_{n a}^{\prime} \Sigma_{n}^{-1} & -\Sigma_{n}^{-1} \boldsymbol{\sigma}_{n a} \Omega^{-1} \\
-\Omega^{-1} \boldsymbol{\sigma}_{n a}^{\prime} \Sigma_{n}^{-1} & \Omega^{-1}
\end{array}\right] \times\left[\begin{array}{c}
\mathbf{1}_{n} \\
1
\end{array}\right] \\
& =\left[\begin{array}{l}
\Sigma_{n}^{-1} \mathbf{1}_{n}+\Sigma_{n}^{-1} \boldsymbol{\sigma}_{n a} \Omega^{-1} \boldsymbol{\sigma}_{n a}^{\prime} \Sigma_{n}^{-1} \mathbf{1}_{n}-\Sigma_{n}^{-1} \boldsymbol{\sigma}_{n a} \Omega^{-1} \\
-\Omega^{-1} \boldsymbol{\sigma}_{n a}^{\prime} \Sigma_{n}^{-1} \mathbf{1}_{n}+\Omega^{-1}
\end{array}\right] \\
& =\left[\begin{array}{l}
\Sigma_{n}^{-1} \mathbf{1}_{n}-\Sigma_{n}^{-1} \boldsymbol{\sigma}_{n a} D-\Sigma_{n}^{-1} \boldsymbol{\sigma}_{n a} \Omega^{-1} \\
D+\Omega^{-1}
\end{array}\right]=\left[\begin{array}{l}
\Sigma_{n}^{-1} \mathbf{1}_{n}-\Sigma_{n}^{-1} \boldsymbol{\sigma}_{n a}\left[D+\Omega^{-1}\right] \\
D+\Omega^{-1}
\end{array}\right]
\end{aligned}
$$

where we define $D \in \mathbb{R}$ as $D \equiv-\Omega^{-1} \boldsymbol{\sigma}_{n a}^{\prime} \Sigma_{n}^{-1} \mathbf{1}_{n}$. 
Compute $A_{(n+1)}=\mathbf{1}_{(n+1)}^{\prime} \Sigma_{(n+1)}^{-1} \mathbf{1}_{(n+1)}$

$$
\begin{aligned}
A_{(n+1)} & =\left[\begin{array}{ll}
\mathbf{1}_{n}^{\prime} & 1
\end{array}\right] \times\left[\begin{array}{l}
\Sigma_{n}^{-1} \mathbf{1}_{n}-\Sigma_{n}^{-1} \boldsymbol{\sigma}_{n a}\left[D+\Omega^{-1}\right] \\
D+\Omega^{-1}
\end{array}\right] \\
& =\mathbf{1}_{n}^{\prime} \Sigma_{n}^{-1} \mathbf{1}_{n}-\mathbf{1}_{n}^{\prime} \Sigma_{n}^{-1} \boldsymbol{\sigma}_{n a}\left[D+\Omega^{-1}\right]+D+\Omega^{-1} \\
& =A_{n}-\mathbf{1}_{n}^{\prime} \Sigma_{n}^{-1} \boldsymbol{\sigma}_{n a}\left[D+\Omega^{-1}\right]+D+\Omega^{-1} \\
& =A_{n}+D \Omega\left[D+\Omega^{-1}\right]+D+\Omega^{-1}=A_{n}+D^{2} \Omega+D+D+\Omega^{-1} \\
& =A_{n}+[1+D \Omega]\left[D+\Omega^{-1}\right]
\end{aligned}
$$

where $\mathbf{1}_{n}^{\prime} \Sigma_{n}^{-1} \boldsymbol{\sigma}_{n a}=-D \Omega$ by the definition of $D$.

Work with $\Sigma_{(n+1)}^{-1} \overline{\mathbf{z}}_{(n+1)}$

$$
\begin{aligned}
\Sigma_{(n+1)}^{-1} \overline{\mathbf{z}}_{(n+1)} & =\left[\begin{array}{ll}
\Sigma_{n}^{-1}+\Sigma_{n}^{-1} \boldsymbol{\sigma}_{n a} \Omega^{-1} \boldsymbol{\sigma}_{n a}^{\prime} \Sigma_{n}^{-1} & -\Sigma_{n}^{-1} \boldsymbol{\sigma}_{n a} \Omega^{-1} \\
-\Omega^{-1} \boldsymbol{\sigma}_{n a}^{\prime} \Sigma_{n}^{-1} & \Omega^{-1}
\end{array}\right] \times\left[\begin{array}{c}
\overline{\mathbf{z}}_{n} \\
\bar{z}_{a}
\end{array}\right] \\
& =\left[\begin{array}{l}
\Sigma_{n}^{-1} \overline{\mathbf{z}}_{n}+\Sigma_{n}^{-1} \boldsymbol{\sigma}_{n a} \Omega^{-1} \boldsymbol{\sigma}_{n a}^{\prime} \Sigma_{n}^{-1} \overline{\mathbf{z}}_{n}-\Sigma_{n}^{-1} \boldsymbol{\sigma}_{n a} \Omega^{-1} \bar{z}_{a} \\
-\Omega^{-1} \boldsymbol{\sigma}_{n a}^{\prime} \Sigma_{n}^{-1} \overline{\mathbf{z}}_{n}+\Omega^{-1} \bar{z}_{a}
\end{array}\right] \\
& =\left[\begin{array}{l}
\Sigma_{n}^{-1} \overline{\mathbf{z}}_{n}-\Sigma_{n}^{-1} \boldsymbol{\sigma}_{n a} E-\Sigma_{n}^{-1} \boldsymbol{\sigma}_{n a} \Omega^{-1} \bar{z}_{a} \\
E+\Omega^{-1} \bar{z}_{a}
\end{array}\right]=\left[\begin{array}{c}
\Sigma_{n}^{-1} \overline{\mathbf{z}}_{n}-\Sigma_{n}^{-1} \boldsymbol{\sigma}_{n a}\left[E+\Omega^{-1} \bar{z}_{a}\right] \\
E+\Omega^{-1} \bar{z}_{a}
\end{array}\right]
\end{aligned}
$$

where we define $E \in \mathbb{R}$ as $E \equiv-\Omega^{-1} \boldsymbol{\sigma}_{n a}^{\prime} \Sigma_{n}^{-1} \overline{\mathbf{z}}_{n}$.

Compute $B_{(n+1)}=\mathbf{1}_{(n+1)}^{\prime} \Sigma_{(n+1)}^{-1} \overline{\mathbf{z}}_{(n+1)}$

$$
\begin{aligned}
B_{(n+1)} & =\left[\begin{array}{ll}
\mathbf{1}_{n}^{\prime} & 1
\end{array}\right] \times\left[\begin{array}{l}
\Sigma_{n}^{-1} \overline{\mathbf{z}}_{n}-\Sigma_{n}^{-1} \boldsymbol{\sigma}_{n a}\left[E+\Omega^{-1} \bar{z}_{a}\right] \\
E+\Omega^{-1} \bar{z}_{a}
\end{array}\right] \\
& =\mathbf{1}_{n}^{\prime} \Sigma_{n}^{-1} \overline{\mathbf{z}}_{n}-\mathbf{1}_{n}^{\prime} \Sigma_{n}^{-1} \boldsymbol{\sigma}_{n a}\left[E+\Omega^{-1} \bar{z}_{a}\right]+E+\Omega^{-1} \bar{z}_{a} \\
& =B_{n}+D \Omega\left[E+\Omega^{-1} \bar{z}_{a}\right]+E+\Omega^{-1} \bar{z}_{a} \\
& =B_{n}+[1+D \Omega]\left[E+\Omega^{-1} \bar{z}_{a}\right]
\end{aligned}
$$

where $\mathbf{1}_{n}^{\prime} \Sigma_{n}^{-1} \boldsymbol{\sigma}_{n a}=-D \Omega$ by the definition of $D$. 
Compute $C_{(n+1)}=\overline{\mathbf{z}}_{(n+1)}^{\prime} \Sigma_{(n+1)}^{-1} \overline{\mathbf{z}}_{(n+1)}$

$$
\begin{aligned}
C_{(n+1)} & =\left[\begin{array}{cc}
\overline{\mathbf{z}}_{n}^{\prime} & \bar{z}_{a}
\end{array}\right] \times\left[\begin{array}{l}
\Sigma_{n}^{-1} \overline{\mathbf{z}}_{n}-\Sigma_{n}^{-1} \boldsymbol{\sigma}_{n a}\left[E+\Omega^{-1} \bar{z}_{a}\right] \\
E+\Omega^{-1} \bar{z}_{a}
\end{array}\right] \\
& =\overline{\mathbf{z}}_{n}^{\prime} \Sigma_{n}^{-1} \overline{\mathbf{z}}_{n}-\overline{\mathbf{z}}_{n}^{\prime} \Sigma_{n}^{-1} \boldsymbol{\sigma}_{n a}\left[E+\Omega^{-1} \bar{z}_{a}\right]+\bar{z}_{a}\left(E+\Omega^{-1} \bar{z}_{a}\right) \\
& =C_{n}+E \Omega\left[E+\Omega^{-1} \bar{z}_{a}\right]+\bar{z}_{a}\left(E+\Omega^{-1} \bar{z}_{a}\right) \\
& =C_{n}+\left[E \Omega+\bar{z}_{a}\right]\left[E+\Omega^{-1} \bar{z}_{a}\right]
\end{aligned}
$$

where $\overline{\mathbf{z}}_{n}^{\prime} \Sigma_{n}^{-1} \boldsymbol{\sigma}_{n a}=-E \Omega$ by the definition of $E$.

Compute $\triangle_{(n+1)}=A_{(n+1)} C_{(n+1)}-\left(B_{(n+1)}\right)^{2}$

$$
\triangle_{(n+1)}=\left\{A_{n}+[1+D \Omega]\left[D+\Omega^{-1}\right]\right\} \times\left\{C_{n}+\left[E \Omega+\bar{z}_{a}\right]\left[E+\Omega^{-1} \bar{z}_{a}\right]\right\}-\left(B_{(n+1)}\right)^{2}
$$

Proof of Theorem 7 (The Main Theorem). For a portfolio in $\mathcal{V}_{(n+1)}$ the optimal weight 
is

$$
\begin{aligned}
\mathbf{w}_{(n+1)}^{*}(\mu)= & \mathbf{w}_{(n+1)}^{*}=\lambda_{(n+1)} \Sigma_{(n+1)}^{-1} \mathbf{1}_{(n+1)}+\gamma_{(n+1)} \Sigma_{(n+1)}^{-1} \overline{\mathbf{z}}_{(n+1)} \\
= & \lambda_{(n+1)}\left[\begin{array}{l}
\Sigma_{n}^{-1} \mathbf{1}_{n}-\Sigma_{n}^{-1} \boldsymbol{\sigma}_{n a}\left[D+\Omega^{-1}\right] \\
D+\Omega^{-1}
\end{array}\right]+\gamma_{(n+1)}\left[\begin{array}{l}
\Sigma_{n}^{-1} \overline{\mathbf{z}}_{n}-\Sigma_{n}^{-1} \boldsymbol{\sigma}_{n a}\left[E+\Omega^{-1} \bar{z}_{a}\right] \\
E+\Omega^{-1} \bar{z}_{a}
\end{array}\right] \\
= & \lambda_{(n+1)}\left\{\left[\begin{array}{l}
\Sigma_{n}^{-1} \mathbf{1}_{n} \\
0
\end{array}\right]+\left(D+\Omega^{-1}\right)\left[\begin{array}{l}
\left.-\Sigma_{n}^{-1} \boldsymbol{\sigma}_{n a}\right] \\
1
\end{array}\right]\right\} \\
& \left.+\gamma_{(n+1)}\left\{\begin{array}{l}
\Sigma_{n}^{-1} \overline{\mathbf{z}}_{n} \\
0
\end{array}\right]+\left(E+\Omega^{-1} \bar{z}_{a}\right)\left[\begin{array}{l}
-\Sigma_{n}^{-1} \boldsymbol{\sigma}_{n a} \\
1
\end{array}\right]\right\} \\
= & \left.\lambda_{(n+1)}\left[\begin{array}{l}
\Sigma_{n}^{-1} \mathbf{1}_{n} \\
0
\end{array}\right]+\gamma_{(n+1)}\left[\begin{array}{l}
\Sigma_{n}^{-1} \overline{\mathbf{z}}_{n} \\
0
\end{array}\right]\right\} \\
& +\left\{\lambda_{(n+1)}\left(D+\Omega^{-1}\right)+\gamma_{(n+1)}\left(E+\Omega^{-1} \bar{z}_{a}\right)\right\}\left[\begin{array}{l}
-\Sigma_{n}^{-1} \boldsymbol{\sigma}_{n a} \\
1
\end{array}\right] \\
= & \lambda_{(n+1)}\left[\begin{array}{l}
A_{n} \mathbf{w}_{n g} \\
0
\end{array}\right]+\gamma_{(n+1)}\left[\begin{array}{l}
B_{n} \mathbf{w}_{n d} \\
0
\end{array}\right] \\
& +\left\{\lambda_{(n+1)}\left(D+\Omega^{-1}\right)+\gamma_{(n+1)}\left(E+\Omega^{-1} \bar{z}_{a}\right)\right\}\left[\begin{array}{l}
-\Sigma_{n}^{-1} \boldsymbol{\sigma}_{n a} \\
1
\end{array}\right]
\end{aligned}
$$

To show that we have a portfolio combination of three mutual funds, we need to show that the weights in front of the mutual funds add up to unity.

$$
\begin{aligned}
& 1 \stackrel{?}{=} \lambda_{(n+1)} A_{n}+\gamma_{(n+1)} B_{n}+(D \Omega+1)\left\{\lambda_{(n+1)}\left(D+\Omega^{-1}\right)+\gamma_{(n+1)}\left(E+\Omega^{-1} \bar{z}_{a}\right)\right\} \\
= & \lambda_{(n+1)} A_{n}+\gamma_{(n+1)} B_{n}+\lambda_{(n+1)}\left[A_{(n+1)}-A_{n}\right]+\gamma_{(n+1)}\left[B_{(n+1)}-B_{n}\right] \\
= & \lambda_{(n+1)} A_{(n+1)}+\gamma_{(n+1)} B_{(n+1)} \\
= & \frac{A_{(n+1)} C_{(n+1)}-A_{(n+1)} B_{(n+1)} \mu_{(n+1)}+A_{(n+1)} B_{(n+1)} \mu_{(n+1)}-B_{(n+1)}^{2}}{\triangle_{(n+1)}}=1,
\end{aligned}
$$

where we have used the relationships between $A_{(n+1)}$ and $A_{n}$, and between $B_{(n+1)}$ and $B_{n}$. We also used the expressions for $\lambda_{(n+1)}$ and $\gamma_{(n+1)}$ and the definition of $\triangle_{n}$. 


\section{References}

[1] Allen, Franklin and Douglas Gale, 1994, Financial Innovation and Risk Sharing. Massachusetts Institute of Technology.

[2] Best, Michael J. and Robert R. Grauer, 1991, "On the Sensitivity of Mean-Variance-Efficient Portfolios to Changes in Asset Means: Some Analytical and Computational Results," Review of Financial Studies 4, 315-342.

[3] Cass, D., and J. Stiglitz, 1970, "The Structure of Investor Preferences and Asset Returns, and Separability in Portfolio Allocation: A Contribution to the Pure Theory of Mutual Funds," Journal of Economic Theory 2.

[4] Chopra, V. K., and W. T. Ziemba, 1993, "The Effect of Errors in Means, Variances, and Covariances on Optimal Portfolio Choice," Journal of Portfolio Management 19, no. 2, 6-11.

[5] Dybvig, Philip H., 1984, "Short Sales Restrictions and Kinks of the Mean Variance Frontier," Journal of Finance 39: 239-44.

[6] Elton, E.J., and M.J. Gruber, 1977, "Risk Reduction and Portfolio Size: An Analytic Solution," Journal of Business 50, 415-37

[7] Engle, C. and A, Rodrigues, 1987, "Tests of International C.A.P.M. with Time Varying Covariances," Report No. 2303. Washington, DC: National Bureau of Economic Research.

[8] Fama, Eugene, 1976, Foundations of finance : portfolio decisions and securities prices. New York : Basic Books.

[9] Feldman, David, and Haim Reisman, 2003, "Simple Construction of the Efficient Frontier," European Financial Management 9, 251-259.

[10] Ferreira, Miguel A. and Paulo M. Gama, 2005, "Have World, Country, and Industry Risks Changed over Time? An Investigation of the Volatility of Developed Stock Markets," Journal of Financial and Quantitative Analysis 40, 195-222. 
[11] Ferson, W., Kandel, S. and R. Stambaugh, 1987, "Tests of Asset Pricing with Time Varying Expected Risk Premiums and Market Betas," Journal of Finance, 42, 201-220.

[12] Giansante, Joseph E., Brian C. Dragun, 2001, "Investment Portfolio Selection System and Method," United States Patent, No.: 6,275,814.

[13] Gibbons, Michael and Wayne Ferson, 1985, "Testing Asset Pricing Models with Changing Expectations and an Unobservable Market Portfolio," Journal of Financial Economics, 14, $217-236$.

[14] Gibbons, M., Ross, S. and Shanken (1989) "A Test of the Efficiency of a Given Portfolio", Econometrica, 57, 1121-1152.

[15] Goetzmann, William N. and Andrey D. Ukhov, 2005, "British Investment Overseas 1870-1913: A Modern Portfolio Theory Approach," NBER Working Paper 11266.

[16] Huberman, G., and S. Kandel, 1987, "Mean Variance Spanning," Journal of Finance, 42, 873-888.

[17] Ingersoll, Jonathan E., (1987). Theory of Financial Decision Making. Rowman \& Littlefield.

[18] Kandel, Shmuel, 1984, "On the Exclusion of Assets from Tests of the Mean Variance Efficiency of the Market Portfolio," Journal of Finance 39, 63-75.

[19] Levy, Haim, and Moshe Levy, 2004, "Prospect Theory and Mean-Variance Analysis," Review of Financial Studies 17, 1015-1041.

[20] Lowenfeld, Henry, 1907, Investment An Exact Science, The Financial Review of Reviews, London.

[21] Markowitz, Harry M., 1952, "Portfolio Selection," The Journal of Finance 7, 77-91.

[22] Markowitz, Harry M., 1991, "Foundations of Portfolio Theory," The Journal of Finance 46, 469-477. 
[23] Merton, R., 1972, "An Analytic Derivation of the Efficient Portfolio Frontier," Journal of Financial and Quantitative Analysis 7, 1851-1872.

[24] Michaud, Richard O., and Robert Michaud, 1999, "Portfolio Optimization by Means of Resampled Efficient Frontiers," United States Patent, No.: 6,003,018.

[25] Miller, Merton H., 1991, Financial Innovations and Market Volatility, Blackwell Publishers, Cambridge, Massachusetts.

[26] Oh, Gyutaeg, 1996, "Some Results in the CAPM with Nontraded Endowments," Management Science 42, 286-293.

[27] Pastor, Lubos, and Robert F. Stambaugh, 2000, "Comparing Asset Pricing Models: An Investment Perspective," Journal of Financial Economics 56, 335-381.

[28] Roll, Richard, 1977, "A Critique Of the Asset Pricing Theory's Tests Part I: On Past and Potential Testability of the Theory,". Journal of Financial Economics 4, 129-176.

[29] Roll, Richard, 1978, "Ambiguity when Performance is Measured by the Securities Market Line," The Journal of Finance 33, 1051-1069.

[30] Scott, Jason S., Christopher L. Jones, James W. Shearer, and John G. Watson, 2001, "Enhancing Utility and Diversifying Model Risk in a Portfolio Optimization Framework," United States Patent, No.: 6,292,787.

[31] Shanken, Jay, 1986, "On the Exclusion of Assets from Tests of the Mean Variance Efficiency of the Market Portfolio: An Extension," Journal of Finance 41, 331-337.

[32] Shefrin, Hersh, and Meir Statman, 1994, "Behavioral Capital Asset Pricing Theory," Journal of Financial and Quantitative Analysis 29, 323-349.

[33] Shefrin, Hersh, and Meir Statman, 2000, "Behavioral Portfolio Theory," Journal of Financial and Quantitative Analysis 35, 127-151. 
[34] Stambaugh, R., 1982, "On the Exclusion of Assets from Tests of the Two Parameters Model: A Sensitivity Analysis," Journal of Financial Economics 10, 237-268.

[35] Statman, Meir, 1987, "How Many Stocks Make a Diversified Portfolio?" Journal of Financial and Quantitative Analysis 22, 353-64.

[36] Ukhov, Andrey D., 2003, “Asset Prices when Investment Opportunities are Changing," Working paper, Indiana University, Kelley School of Business.

[37] Vygodsky, M., (1975). Mathematical Handbook: Higher Mathematics. Moscow: Mir Publishers. 


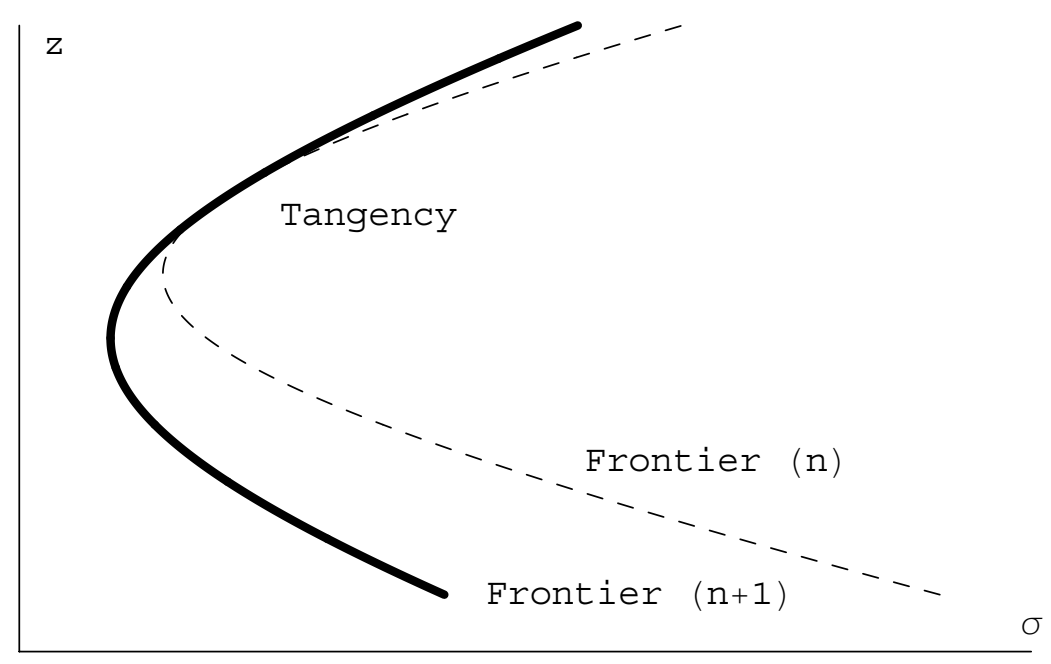

Figure 1: Minimum variance frontiers generated with $n$ and $(n+1)$ assets. The tangency point is above the global minimum variance portfolio. Parameter values: Standard Deviations are $\sigma_{1}=0.15$, $\sigma_{2}=0.20, \sigma_{3}=0.23 ;$ Correlations are $\rho_{12}=0.1, \rho_{13}=0.25, \rho_{23}=0.0 ;$ Expected returns are $z_{1}=1.16, z_{2}=1.18, z_{3}=1.145$.

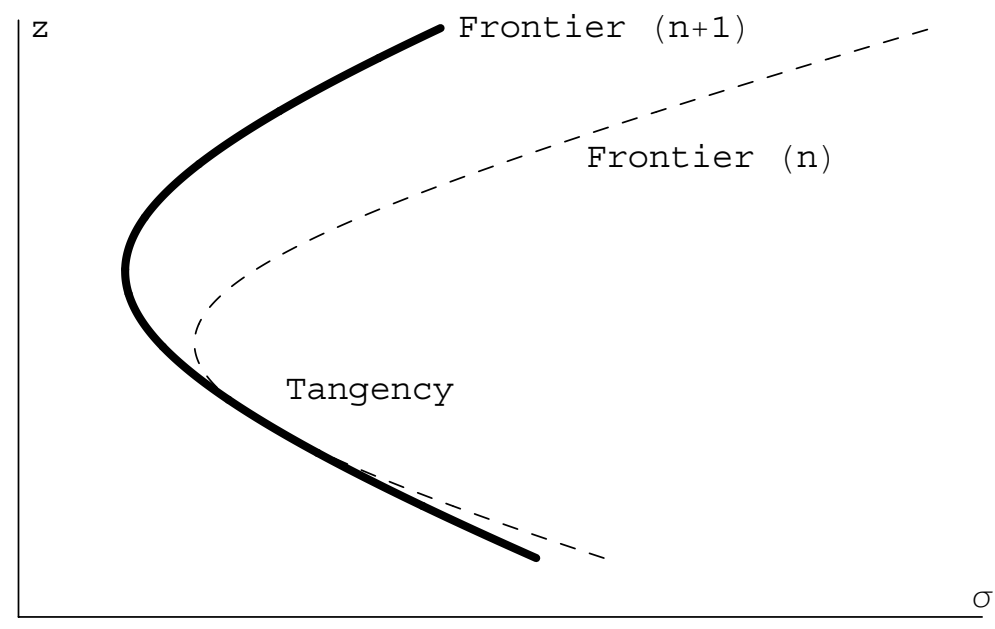

Figure 2: Minimum variance frontiers generated with $n$ and $(n+1)$ assets. The tangency point is below the global minimum variance portfolio. Parameter values: Standard Deviations are: $\sigma_{1}=$ 0.15, $\sigma_{2}=0.35, \sigma_{3}=0.9$; Correlations are $\rho_{12}=0.2, \rho_{13}=0.5, \rho_{23}=0.45$; Expected returns are $z_{1}=1.1, z_{2}=1.2, z_{3}=0.95$. 